\title{
Directed forgetting in young children: Evidence for a production deficiency
}

\author{
Alp Aslan, Tobias Staudigl, Anuscheh Samenieh, and Karl-Heinz T. Bäuml \\ Regensburg University, Regensburg, Germany
}

\begin{abstract}
When people are cued to forget a previously studied list of items and to learn a new list instead, such cuing typically leads to forgetting of the first list and to memory enhancement of the second. In two experiments, we examined such listwise directed forgetting in children (and adults), using a forget cue that placed either high emphasis or low emphasis on the need to forget. In the low-emphasis condition, (adult-like) List 1 forgetting was present in fourth graders, but not in first graders (and kindergartners); in contrast, in the high-emphasis condition, (adult-like) List 1 forgetting was present from first grade on. Only fourth graders showed (adult-like) List 2 enhancement, regardless of task instruction. The finding that first graders showed List 1 forgetting only in the high-emphasis condition points to a production deficiency in first graders' directed forgetting, suggesting that the children are capable of intentional forgetting but fail to do so spontaneously. The finding that first graders showed List 1 forgetting without List 2 enhancement suggests that the two directed-forgetting effects are mediated by different processes with different developmental trajectories.
\end{abstract}

We are all aware of situations in which we would wish to forget previously stored information, be it because the information is emotionally straining, like a past traumatic experience, or because the information is outdated and may interfere with currently more relevant information, like an expired computer password. Laboratory work on directed forgetting (DF) has revealed that people can indeed intentionally forget information when they wish to do so. In listwise DF, subjects study two lists of items. After study of the first list, they receive a cue to forget the list and to learn a new, second list instead. Following study of the second list, a recall test is conducted in which subjects are asked to recall all of the previously presented items, including those they were originally cued to forget. Compared with subjects cued to remember both lists of items, forget-cued subjects typically show improved recall of the second list (List 2 enhancement); in particular, they show impaired recall of the first list (List 1 forgetting) that is, efficient intentional forgetting (for reviews, see MacLeod, 1998, or Bäuml, 2008).

Two prominent accounts of DF are retrieval inhibition and context change. The retrieval inhibition account assumes that forget-cued subjects engage in active inhibitory processes on the to-be-forgotten material; the inhibition reduces accessibility of List 1 and, due to the resulting decrease in these items' interference potential, simultaneously facilitates memory for List 2 (Geiselman, Bjork, \& Fishman, 1983). The context-change account assumes that subjects deliberately change their internal context in response to the forget cue. Such change in internal context leads to a mismatch between the encoding and retrieval contexts and thus to context-dependent forgetting of List 1; simultaneously, the context change reduces proactive interference from List 1 and thus improves recall of List 2 (Sahakyan \& Kelley, 2002).

Previous developmental work has found that young children show difficulties in the DF task (for a review, see Wilson \& Kipp, 1998). Harnishfeger and Pope (1996), for instance, examined first, third, and fifth graders and young adults. Whereas fifth graders showed intact (adultlike) List 1 forgetting, the forgetting was reduced in third graders and was completely absent in first graders. Similarly, examining second and fourth graders, Zellner and Bäuml (2004) found reliable DF in the older children, but no effects of the forget cue in the younger children. These findings indicate that intentional forgetting is a late-emerging memorial capability that reaches maturity not before the end of the elementary school years. In particular, the findings suggest that the processes that mediate DF in adults are deficient in younger elementary school children. Although prevalent in the developmental literature (e.g., Harnishfeger \& Pope, 1996), such a (mediational-deficiency) view might be premature.

Indeed, previous developmental research has shown that when children are acquiring a new mnemonic skill, there is often a transitional period during which the children are capable of executing the skill but fail to do so spontaneously, unless they are appropriately instructed. Such a period of production deficiency has consistently been found in research on memory strategy development (for a review, see Schneider \& Pressley, 1997) and has been reported for children's use of rehearsal strategies

A. Aslan, alp.aslan@psychologie.uni-regensburg.de 
(Flavell, 1970), organization strategies (Ornstein \& Corsale, 1979), and elaboration strategies (Pressley, 1982). Regarding young children's DF, a production deficiency could arise because DF depends on effortful and resourcedemanding processes (see, e.g., Aslan, Zellner, \& Bäuml, 2010; Conway, Harries, Noyes, Racsma'ny, \& Frankish, 2000). In fact, previous developmental work showed that younger children are often less willing than older children to engage in effortful (though available) mnemonic processes, whose execution places high demands on their limited mental resources (e.g., Guttentag, 1984).

There is some indirect evidence for the view that mature $\mathrm{DF}$ is preceded by a period of production deficiency. Aslan and Bäuml (2008), for instance, examined the memorial effects of an internal context change in children and adults. In contrast to the DF procedure, the context change was not left to the subject's initiative, but rather was induced experimentally by asking subjects to imagine being invisible. Aslan and Bäuml (2008) found adult-like context-dependent forgetting in first and fourth graders (though not in kindergartners), indicating that, by first grade, children can change their internal context when urged to do so. Also, several recent studies examined children's inhibitory capabilities in episodic memory using tasks other than DF. These studies reported adult-like retrieval-induced and part-list-cuing inhibition in children as young as first graders (e.g., Aslan \& Bäuml, 2010; Zellner \& Bäuml, 2005). These findings indicate that both inhibitory and context-change processes in children's episodic memory may be intact in first graders. Following the view that DF is mediated by inhibitory or context-change processes, young children's failure to show DF thus might reflect a deficiency to spontaneously engage in available processes, rather than a deficiency in the processes themselves.

The present study addressed the issue directly by examining whether DF can be triggered in young children with appropriate task instruction. In particular, we manipulated the wording of the forget cue, contrasting two forget instructions that have often been used in adult studies. The one instruction (sometimes called the "whoops" procedure; see the Method section) provided a comprehensible rationale for the (unexpected) forget cue and placed high emphasis on the need to forget (high-emphasis condition); the other instruction did not provide a rationale for the forget cue and emphasis on the need to forget was relatively low (low-emphasis condition). On the basis of previous work (e.g., Sahakyan, Delaney, \& Goodmon, 2008), we expected that the wording of the forget instruction would not affect young adults' performance, and that adults would show reliable DF in both the high-emphasis and the low-emphasis conditions. If young children suffered from a fundamental deficiency in the required processes, efficient DF should be absent in both the high-emphasis and low-emphasis condition. Alternatively, if young children suffered from a production deficiency - that is, if they had the required processes available but did not engage in these processes spontaneously-DF should be absent in the low-emphasis condition but might be present in the high-emphasis condition.
To date, there is only one study in which the question of whether mature DF is preceded by a period of production deficiency has already been addressed. Providing first graders with a graphical model that supposedly conveyed (metacognitive) insight into the usefulness of forgetting, Hasselhorn and Richter (2002) found significant DF in first graders. However, in marked contrast to the literature (see Wilson \& Kipp, 1998), first graders also showed forgetting when no insight into the usefulness of forgetting was provided, thus leaving it open whether young children's (typically observed) failure to show efficient DF reflects a production deficiency.

\section{EXPERIMENT 1}

\section{Method}

Subjects. Fifty-six kindergartners $(M=4.6$ years, $S D=0.5)$, 56 first graders $(M=6.7$ years, $S D=0.5), 56$ fourth graders $(M=$ 9.8 years, $S D=0.4)$, and 56 young adults $(M=23.5$ years, $S D=$ 3.5) participated in the experiment. The children were recruited from several kindergartens and elementary schools in Regensburg, Germany; the adults were students at Regensburg University. All subjects were tested individually.

Materials. Four study lists were constructed, each list consisting of six unrelated items drawn from a German norm for children (Hasselhorn, Jaspers, \& Hernando, 1990; Posnansky, 1978).

Design and Procedure. The experiment had a mixed design with the between-subjects factors of age group (kindergartners, first graders, fourth graders, adults) and task instruction (low emphasis, high emphasis) and the within-subjects factor of cue (remember, forget). For each subject, the experiment consisted of two parts that differed in the cue that was provided. In each of the two parts, subjects were read two lists of items at a $3-\mathrm{sec}$ rate in random order. Between the two lists, the interlist cue was provided. In the remember condition, subjects were told that the preceding items were the first part of the study list and should be kept in mind while they studied the second part; this held for subjects in both the low-emphasis and the highemphasis groups. In contrast, the forget condition differed between the low-emphasis and the high-emphasis groups. In the low-emphasis group, subjects were told that the preceding items could be forgotten because they would not be tested later. In the high-emphasis group, the experimenter became flustered after presentation of List 1 and pretended that she had made a mistake and presented a wrong list. She apologized and asked the subject emphatically to try his/her best to forget those "incorrect" items and to concentrate on the following list of items, which would be the correct one. After presentation of the second list and a 90-sec distractor (counting) task, a recall test for all items was conducted. Subjects were asked to recall List 1 items first and List 2 items second. Subjects had 1 min per list but were given extra time when needed. The verbal responses were noted by the experimenter. After a 3-min break, the second part of the experiment with the second cue condition started. The order of the remember and the forget condition was counterbalanced across subjects, as was the assignment of lists to cue (remember or forget) and list position (List 1 or List 2).

\section{Results}

Following prior work (e.g., Pastötter \& Bäuml, 2010; Sahakyan \& Kelley, 2002), we analyzed List 1 and List 2 recall separately. ${ }^{1}$

Regarding List 1 recall (upper panel of Figure 1), a $2 \times 4 \times 2$ ANOVA with the factors of cue (remember, forget), age group (kindergartners, first graders, fourth graders, adults), and task instruction (low emphasis, high emphasis) revealed significant main effects of cue 


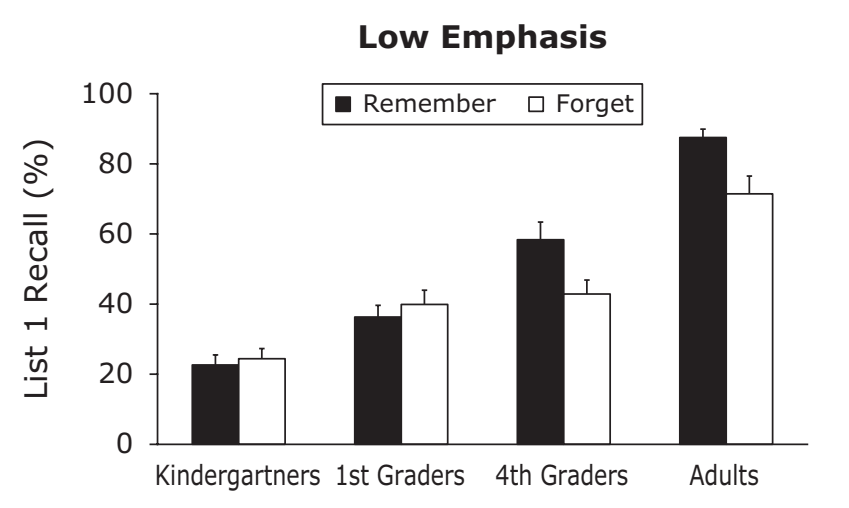

Low Emphasis

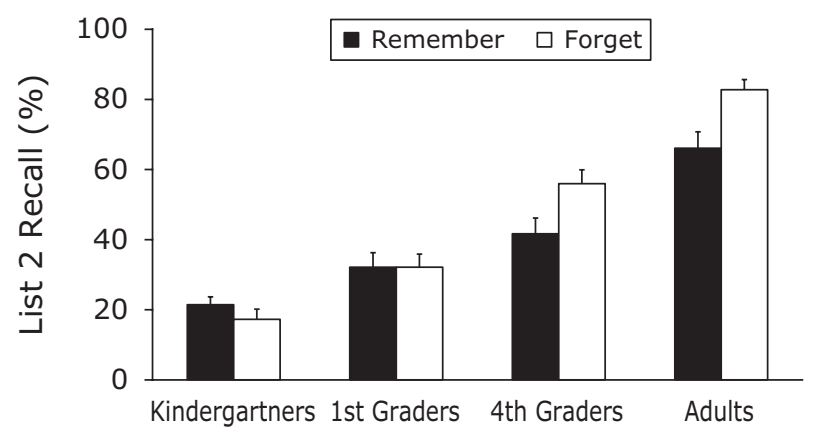

High Emphasis

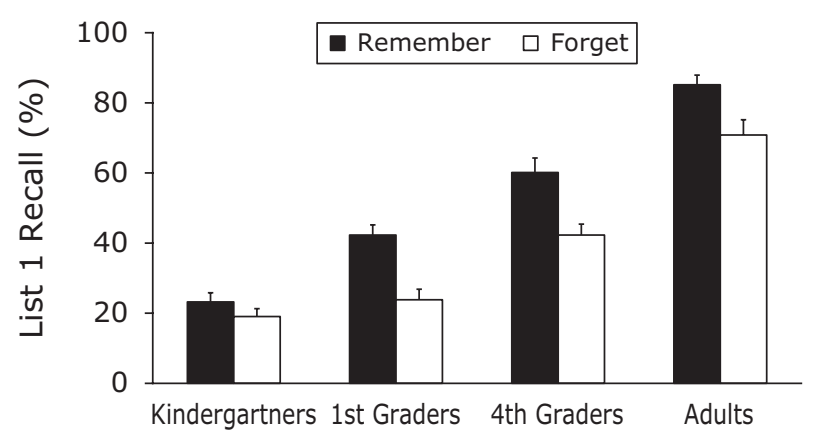

High Emphasis

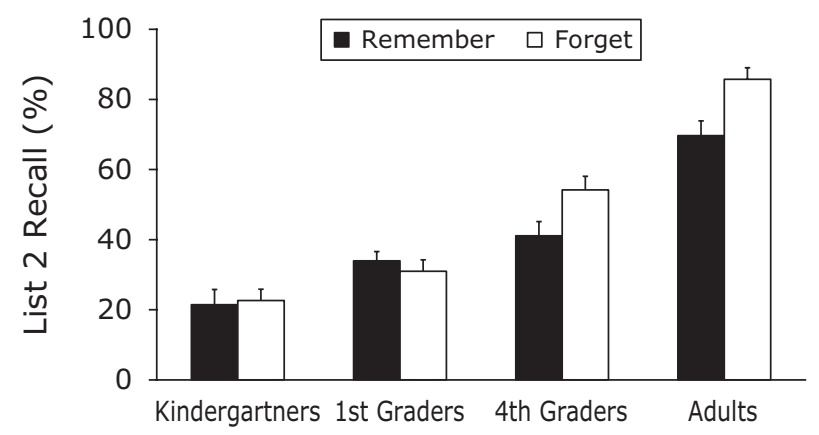

Figure 1. Experiment 1: Recall performance for List 1 (top) and List 2 (bottom) in the low-emphasis (left) and the high-emphasis (right) conditions as a function of cue (remember, forget) and age group (kindergartners, first graders, fourth graders, adults). The error bars represent standard errors.

$\left[F(1,216)=44.7, M S_{\mathrm{e}}=0.026, p<.001\right]$ and age group $\left[F(3,216)=149.1, M S_{\mathrm{e}}=0.044, p<.001\right]$, but no main effect of task instruction $\left[F(1,216)=1.1, M S_{\mathrm{e}}=0.044\right.$, $p>$.20]. The significant main effects reflect reduced overall recall in the forget compared with the remember condition, and higher overall recall in older compared with younger subjects ( $p$ s $<.001$ for all pairwise comparisons). There were also significant two-way interactions between cue and age group $\left[F(3,216)=5.6, M S_{\mathrm{e}}=\right.$ $0.026, p=.001]$ and cue and task instruction $[F(1,216)=$ $\left.5.6, M S_{\mathrm{e}}=0.026, p<.05\right]$. Most important in the present context, there was a significant three-way interaction $\left[F(3,216)=3.0, M S_{\mathrm{e}}=0.026, p<.05\right]$, indicating that the developmental course of List 1 forgetting was affected by task instruction. Indeed, whereas in the low-emphasis condition, List 1 forgetting was present in adults and fourth graders (both $p \mathrm{~s}<.01$ ), but not in first graders and kindergartners (both $p \mathrm{~s}>.50$ ), in the high-emphasis condition, List 1 forgetting was present in adults, fourth graders, and first graders (all $p \mathrm{~s}<.01$ ), but not in kindergartners $(p>.20)$.

An analogous ANOVA on List 2 recall (lower panel of Figure 1) revealed significant main effects of cue $\left[F(1,216)=14.6, M S_{\mathrm{e}}=0.035, p<.001\right]$ and age group $\left[F(3,216)=157.0, M S_{\mathrm{e}}=0.041, p<.001\right]$, but no main effect of task instruction $[F(1,216)<1]$. The significant main effects reflect higher overall recall in the forget than in the remember condition, and higher overall recall in older than in younger subjects $(p s<.001$, for all pairwise comparisons). There was also a significant two-way interaction between cue and age group $[F(3,216)=7.3$, $\left.M S_{\mathrm{e}}=0.035, p<.001\right]$. However, there was no reliable three-way interaction $[F(3,216)<1]$, indicating that, in contrast to List 1 forgetting, the developmental course of List 2 enhancement was unaffected by task instruction. Consistently, in both the low-emphasis and high-emphasis conditions, reliable List 2 enhancement was present in adults and fourth graders (all $p \mathrm{~s}<.05$ ) but was absent in first graders and kindergartners (all $p \mathrm{~s}>.20$ ).

\section{Discussion}

In the low-emphasis condition, we found efficient (adult-like) List 1 forgetting in older (fourth grade) children, but no forgetting in younger (first grade and kindergarten) children, thus basically replicating the results of previous studies (Harnishfeger \& Pope, 1996; Zellner \& Bäuml, 2004). In contrast, in the high-emphasis condition, we found reliable forgetting from first grade on. These results demonstrate that young children's DF depends on task instruction, and that even first graders can show successful intentional forgetting when appropriately cued.

Although first graders showed reliable List 1 forgetting in the high-emphasis condition, they did not show any memory improvement for List 2, suggesting that List 2 enhancement emerges later in development than List 1 forgetting. However, in Experiment 1, we asked subjects to 
recall List 1 before List 2. Although this procedure should have revealed relatively pure measures of List 1 forgetting, the prior recall of List 1 items might have contaminated the recall of List 2 items and, in this way, might have masked a possible enhancement effect in first graders (for possible recall order effects in DF, see Golding \& Gottlob, 2005). To address the issue, and replicate the findings of Experiment 1, in Experiment 2 we asked subjects to start their recall with List 2 items. As another difference, we examined first and fourth graders only. Kindergartners and adults were skipped because, in Experiment 1, kindergartners failed to show DF even in the high-emphasis condition, and fourth graders showed essentially the same pattern of results as the adults.

\section{EXPERIMENT 2}

\section{Method}

Subjects. Forty-eight first graders $(M=6.5$ years, $S D=0.5)$, and 48 fourth graders $(M=9.7$ years, $S D=0.5)$, recruited from two elementary schools in Regensburg, Germany, participated in the experiment. They were tested individually.

Materials. The same item materials as in Experiment 1 were used.

Design and Procedure. Design and procedure were identical to those of Experiment 1, except that List 2 was tested before List 1.

\section{Results}

Regarding List 1 recall (upper panel of Figure 2), a $2 \times 2 \times 2$ ANOVA with the factors of cue (remember, for- get), age group (first graders, fourth graders), and task instruction (low emphasis, high emphasis) revealed significant main effects of cue $\left[F(1,92)=24.9, M S_{\mathrm{e}}=0.025\right.$, $p<.001]$, age group $\left[F(1,92)=30.3, M S_{\mathrm{e}}=0.030, p<\right.$ $.001]$, and task instruction $\left[F(1,92)=4.1, M S_{\mathrm{e}}=0.030\right.$, $p<.05]$. These main effects reflect reduced overall recall in the forget condition as compared with in the remember condition, higher overall recall in fourth graders than in first graders, and higher overall recall in the low-emphasis than in the high-emphasis condition. Although none of the two-way interactions reached significance (all $p \mathrm{~s}>.20$ ), a reliable three-way interaction emerged $[F(1,92)=4.3$, $\left.M S_{\mathrm{e}}=0.025, p<.05\right]$, indicating that the developmental course of List 1 forgetting was affected by task instruction. Indeed, whereas in the low-emphasis condition, List 1 forgetting was present only in the fourth graders $(p<.001)$ and not the first graders $(p>.70)$, in the high-emphasis condition, List 1 forgetting was present in both age groups (both $p \mathrm{~s}<.05$ ).

An analogous ANOVA on List 2 recall (lower panel of Figure 2) revealed significant main effects of cue $\left[F(1,92)=15.8, M S_{\mathrm{e}}=0.020, p<.001\right]$ and age group $\left[F(1,92)=51.9, M S_{\mathrm{e}}=0.031, p<.001\right]$, but no main effect of task instruction $\left[F(1,92)=1.4, M S_{\mathrm{e}}=0.031, p>\right.$ .20]. The significant main effects reflect higher overall recall in the forget than in the remember condition, and higher overall recall in fourth graders than in first graders. There was also a significant two-way interaction be-

\section{Low Emphasis}

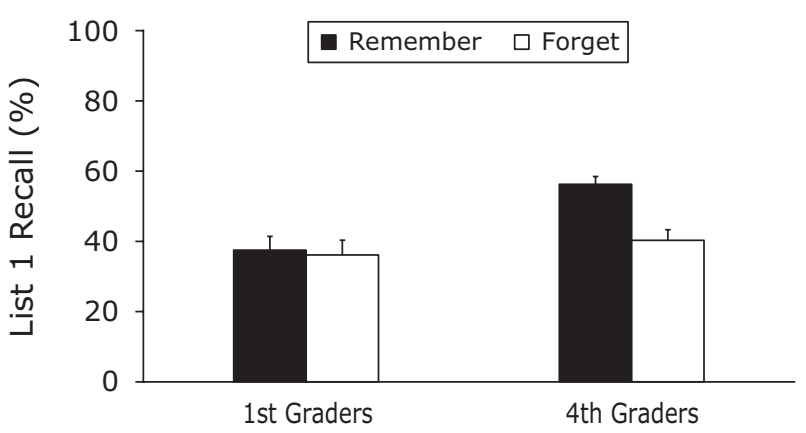

Low Emphasis

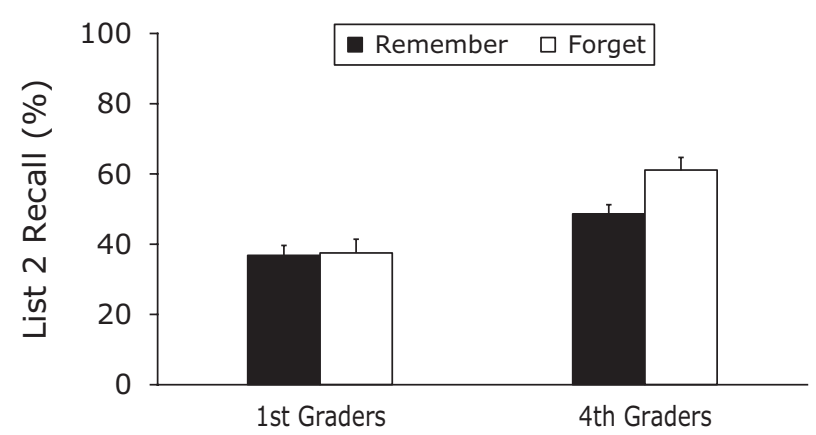

High Emphasis

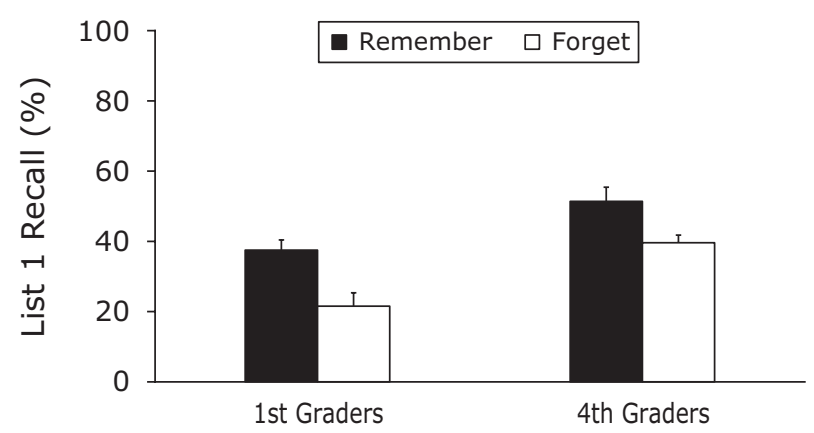

High Emphasis

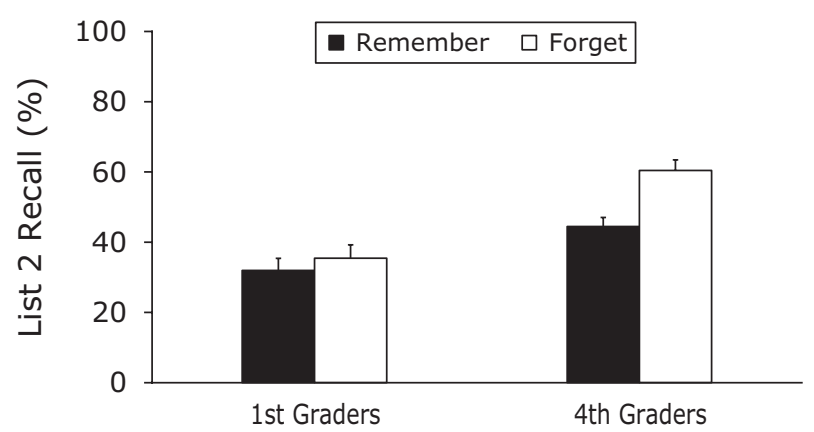

Figure 2. Experiment 2: Recall performance for List 1 (top) and List 2 (bottom) in the low-emphasis (left) and the high-emphasis (right) conditions as a function of cue (remember, forget) and age group (first graders, fourth graders). The error bars represent standard errors. 
tween cue and age group $\left[F(1,92)=8.7, M S_{\mathrm{e}}=0.020\right.$, $p<.005]$. However, as in Experiment 1, no reliable threeway interaction arose $[F(1,92)<1]$, indicating that the developmental course of List 2 enhancement was unaffected by task instruction. Consistently, in both the lowemphasis and high-emphasis conditions, reliable List 2 enhancement was present only in the fourth graders (both $p \mathrm{~s}<.01$ ), but not the first graders (both $p \mathrm{~s}>.40$ ).

\section{Discussion}

Whereas fourth graders showed reliable List 1 forgetting regardless of task instruction, first graders showed forgetting in the high-emphasis, but not the low-emphasis condition. These results replicate the finding of Experiment 1 that first graders can show successful intentional forgetting, at least when appropriately instructed. Also replicating Experiment 1, task instruction did not affect List 2 enhancement, neither its presence in fourth graders nor its absence in first graders. Because in this experiment List 2 items were tested before List 1 items, relatively pure measures of List 2 enhancement should have resulted. The finding of no effect of task instruction on List 2 enhancement thus supports the view that List 2 enhancement emerges later in development than does List 1 forgetting.

\section{GENERAL DISCUSSION}

In two experiments, we examined the role of task instruction in children's DF. When the instruction did not provide a rationale for the (unexpected) forget cue and emphasis on the need to forget was relatively low, we found reliable (adult-like) List 1 forgetting in fourth graders, but no effects of the forget cue in first graders and kindergartners, thus basically replicating results from previous developmental work (e.g., Harnishfeger \& Pope, 1996). In contrast, when the forget instruction provided a comprehensible rationale for the forget cue and placed high emphasis on the need to forget, (adult-like) List 1 forgetting was present from first grade on, in both experiments.

Previous developmental work suggests that children pass through (at least) two kinds of deficiencies before being able to use a memory strategy in an effective and adult-like manner. During the first, mediational-deficiency stage (Reese, 1962), children are not capable of executing the strategy, even with instruction and/or extensive training. During the second, production-deficiency stage (Flavell, 1970), children still do not show the strategy spontaneously, but they are now capable of using the strategy effectively when appropriately instructed. Following this distinction, the present results point to a mediational deficiency in kindergartners' DF. In contrast, the finding that first graders benefited from an appropriate task instruction and showed DF in the high-emphasis, but not in the low-emphasis, condition points to a production deficiency in first graders' DF. These findings indicate that mnemonic processes that lead to successful intentional forgetting follow the same successive stages during development as do the more commonly investigated processes that lead to successful remembering- that is, rehearsal, organization, and elaboration strategies (Schneider \& Pressley, 1997).

Although there may be an infinity of different ways to provide the forget cue, which may all differ in their effectiveness at triggering subjects' engagement in forgetting attempts, in the present study, we made an effort to choose two instructions that covered the range reasonably well. Specifically, we used one instruction that provided a comprehensible rationale for the forget cue and placed high emphasis on the need to forget and one instruction that did not provide such a rationale and emphasized the need to forget to a much lesser extent. Previous work that examined DF in children used forget instructions that did not place much emphasis on the need to forget and thus were more similar to the present low-emphasis condition. For instance, subjects were told that List 1 items could be forgotten because they were "just for practice" (Harnishfeger \& Pope, 1996) or were "only imagined" by a child (Zellner \& Bäuml, 2004). Consistent with the present results, these previous studies failed to find DF in first or second graders.

Although older people generally show efficient DF (e.g., Zellner \& Bäuml, 2006), Sahakyan et al. (2008) recently found reduced DF in older adults who felt that efforts to forget were needless because they forgot List 1 anyway. When the forget instruction was modified to downplay older adults' concerns about their poor memory, older adults were more likely to engage in forgetting attempts and showed DF that was indistinguishable from that of younger adults. These results bear some resemblance to the present findings, indicating that age-related differences in DF may be diminished when age-related differences in individuals' reluctance to engage in forgetting attempts are compensated. However, despite the resemblance, only children's but not older adults' reduced DF may reflect a production deficiency.

Prominent accounts of DF, like retrieval inhibition or context change, assume that the two DF effects are mediated by the same mechanism and thus should always occur concurrently. Although the results of the present low-emphasis condition are consistent with such singlemechanism accounts, neither inhibition nor context change can explain why first graders showed reliable List 1 forgetting but did not show any List 2 enhancement in the highemphasis condition. However, this developmental dissociation is consistent with other work reporting dissociations between the two DF effects (e.g., Bäuml, Hanslmayr, Pastötter, \& Klimesch, 2008; Pastötter \& Bäuml, 2010; Sahakyan \& Delaney, 2003, 2005), and it is consistent with two-mechanism accounts of DF, according to which a retrieval-based mechanism (e.g., inhibition or context change) underlies List 1 forgetting and an encoding-based mechanism (e.g., a change in encoding strategy) underlies List 2 enhancement (Bäuml et al., 2008; Sahakyan \& Delaney, 2003). Following such two-mechanism accounts, the present results suggest that the retrieval-based List 1 mechanism develops earlier than the encoding-based List 2 mechanism and that only the former and not the latter is sensitive to variations in task instruction. 


\section{AUTHOR NOTE}

This work was supported by a grant from the German Research Foundation (DFG) to K.-H.T.B. and A.A. (BA 1382/8-1). We thank A. Fenk and F. X. Weindler for their help with data collection. Correspondence concerning this article should be addressed to A. Aslan, Department of Experimental Psychology, Regensburg University, 93040 Regensburg, Germany (e-mail: alp.aslan@psychologie.uni-regensburg.de).

$$
\text { Note-Accepted by Cathleen M. Moore's editorial team. }
$$

\section{REFERENCES}

Aslan, A., \& BäUmL, K.-H. (2008). Memorial consequences of imagination in children and adults. Psychonomic Bulletin \& Review, 15, 833-837.

Aslan, A., \& BäUML, K.-H. T. (2010). Retrieval-induced forgetting in young children. Psychonomic Bulletin \& Review, 17, 704-709.

Aslan, A., Zellner, M., \& BäUmL, K.-H. T. (2010). Working memory capacity predicts listwise directed forgetting in adults and children. Memory, 18, 442-450.

BäUML, K.-H. (2008). Inhibitory processes. In H. L. Roediger III (Ed.), Learning and memory: A comprehensive reference. Vol. 2: Cognitive psychology of memory (pp. 195-220). Amsterdam: Elsevier.

Bäuml, K.-H., Hanslmayr, S., Pastötter, B., \& Klimesch, W. (2008). Oscillatory correlates of intentional updating in episodic memory. NeuroImage, 41, 596-604.

Conway, M. A., Harries, K., Noyes, J., Racsma'ny, M., \& FrankISH, C. (2000). The disruption and dissolution of directed forgetting: Inhibitory control of memory. Journal of Memory \& Language, 43, 409-430.

FlavelL, J. H. (1970). Developmental studies of mediated memory. In H. W. Reese \& L. P. Lipsitt (Eds.), Advances in child development and behavior (pp. 181-211). New York: Academic Press.

Geiselman, R. E., Buork, R. A., \& Fishman, D. (1983). Disrupted retrieval in directed forgetting: A link with posthypnotic amnesia. Journal of Experimental Psychology: General, 112, 58-72.

Golding, J. M., \& GotTLOB, L. R. (2005). Recall order determines the magnitude of directed forgetting in the within-participants list method. Memory \& Cognition, 33, 588-594.

Guttentag, R. E. (1984). The mental effort requirement of cumulative rehearsal: A developmental study. Journal of Experimental Child Psychology, 37, 92-106.

Harnishfeger, K. K., \& Pope, R. S. (1996). Intending to forget: The development of cognitive inhibition in directed forgetting. Journal of Experimental Child Psychology, 62, 292-315.

Hasselhorn, M., Jaspers, A., \& Hernando, M.-D. (1990). Typizitätsnormen zu zehn Kategorien von der Vorschule bis zur vierten Grundschulklasse. Sprache \& Kognition, 9, 92-108.

Hasselhorn, M., \& Richter, M. (2002). Entwicklung effektiver Abrufhemmung bei Grundschulkindern: Zum Einfluss von Motivation und Einsicht. Zeitschrift für Entwicklungspsychologie und Pädagogische Psychologie, 34, 149-155.
MacLeod, C. M. (1998). Directed forgetting. In J. M. Golding \& C. M. MacLeod (Eds.), Intentional forgetting: Interdisciplinary approaches (pp. 1-57). Mahwah, NJ: Erlbaum.

Ornstein, P. A., \& Corsale, K. (1979). Organizational factors in children's memory. In C. R. Puff (Ed.), Memory, organization, and structure (pp. 69-99). New York: Academic Press.

PastötTer, B., \& BäUML, K.-H. (2010). Amount of postcue encoding predicts amount of directed forgetting. Journal of Experimental Psychology: Learning, Memory, \& Cognition, 36, 54-65.

Posnansky, C. J. (1978). Category norms for verbal items in 25 categories for children in Grades 2-6. Behavior Research Methods \& Instrumentation, 10, 819-832.

PressLey, M. (1982). Elaboration and memory development. Child Development, 53, 296-309.

Reese, H. W. (1962). Verbal mediation as a function of age level. Psychological Bulletin, 59, 502-509.

SahaKyan, L., \& Delaney, P. F. (2003). Can encoding differences explain the benefits of directed forgetting in the list method paradigm? Journal of Memory \& Language, 48, 195-206.

Sahakyan, L., \& Delaney, P. F. (2005). Directed forgetting in incidental learning and recognition testing: Support for a two-factor account. Journal of Experimental Psychology: Learning, Memory, \& Cognition, 31, 789-801.

Sahakyan, L., Delaney, P. F., \& Goodmon, L. B. (2008). Oh, honey, I already forgot that: Strategic control of directed forgetting in older and younger adults. Psychology \& Aging, 23, 621-633.

Sahakyan, L., \& Kelley, C. M. (2002). A contextual change account of the directed forgetting effect. Journal of Experimental Psychology: Learning, Memory, \& Cognition, 28, 1064-1072.

SCHNeIder, W., \& Pressley, M. (1997). Memory development between two and twenty. Mahwah, NJ: Erlbaum.

WILSON, S. P., \& KIPP, K. (1998). The development of efficient inhibition: Evidence from directed-forgetting tasks. Developmental Review, 18, 86-123.

Zellner, M., \& BäUML, K.-H. (2004). Retrieval inhibition in episodic recall. In A. Mecklinger, H. Zimmer, \& U. Lindenberger (Eds.), Bound in memory: Insights from behavioral and neuropsychological studies (pp. 1-16). Aachen: Shaker.

ZELLNER, M., \& BäUML, K.-H. (2005). Intact retrieval inhibition in children's episodic recall. Memory \& Cognition, 33, 396-404.

ZeLLnER, M., \& BäUML, K.-H. (2006). Inhibitory deficits in older adults: List-method directed forgetting revisited. Journal of Experimental Psychology: Learning, Memory, \& Cognition, 32, 290-300.

\section{NOTE}

1. In both experiments, counterbalancing did not affect the overall pattern of results (all $p \mathrm{~s}>.05$ ), which is consistent with previous DF work (e.g., Zellner \& Bäuml, 2006)

(Manuscript received March 9, 2010; revision accepted for publication May 21, 2010.) 